\title{
Subkutantherapie: weniger Symptome trotz weniger Medikamenten
}

ängst nicht alle Patienten mit aller- gischer Rhinitis oder allergischem Asthma lassen sich mit einer Pharmakotherapie erfolgreich behandeln. Nur $33 \%$ bzw. $40 \%$ sind damit zufrieden, erläuterte Prof. Dr. Randolf Brehler, Münster. Als Alternative nannte er die subkutane Immuntherapie (SCIT). Er verwies auf eine vergleichende Analyse auf der Basis publizierter Metaanalysen, die Studien zur Wirksamkeit von SCIT, nasalen Glukokortikoiden, Leukotrienantagonisten und Antihistaminika bei saisonaler allergischer Rhinitis berücksichtigten: Die SCIT erwies sich als der symptomatischen Pharmakotherapie überlegen mit einer statistisch signifikant stärkeren Reduktion des GesamtNasen-Symptom-Scores und des Gesamt-Symptom-Scores ( $\mathrm{p}<0,00001)$.

Gleichzeitig lassen sich mit der SCIT Medikamente einsparen, wie eine plazebokontrollierte Studie mit Allergovit $^{\circledR}$ Gräser belegt. In der Studie besserten sich nicht nur die allergischen Beschwerden, auch der Medikationsscore ging um $69 \%$ zurück. Brehler be- tonte zudem die dauerhafte Wirksamkeit der SCIT, die im Verlauf der dreijährigen Therapie stetig zunimmt. Dass die SCIT nachhaltig wirkt, dokumentiert eine Langzeituntersuchung bei Kindern über einen Zeitraum von zwölf

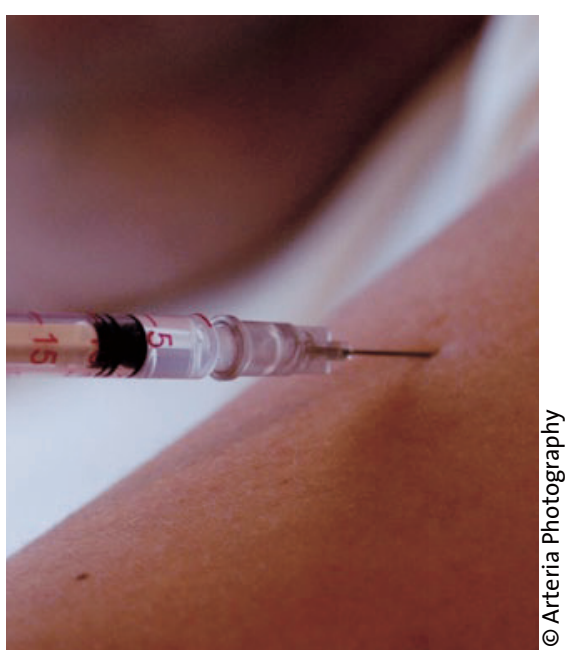

Den Nadelstich macht der direkte Draht zwischen Arzt und Patient bei der SCIT wett: Die subkutane Therapie schneidet auch bei der Compliance gut ab.
Jahren nach Beendigung der SCIT. Danach war die SCIT anhaltend wirksam und reduzierte das Risiko für Asthma und Neusensibilisierungen.

Hohe Wirksamkeit zeigt die SCIT auch bei Kindern mit allergischem Asthma, ausgelöst durch eine Hausstaubmilbenallergie. Hier konnte nach Brehler die zur Asthmakontrolle notwendige Fluticasondosis reduziert werden, während sich gleichzeitig die Lungenfunktion verbesserte.

Entscheidend für den Erfolg der SCIT ist die Compliance. Immerhin müssen die Patienten drei Jahre bei der Stange gehalten werden. Hier präsentierte PD Dr. Oliver Pfaar, Wiesbaden, aktuelle Daten, nach denen die Compliance bei der SCIT deutlich besser ist als bei der sublingualen Immuntherapie (SLIT) und bei perennialer Therapie besser als bei präsaisonaler Therapie. Die jeweils stärkere Interaktion zwischen Arzt und Patient scheint dabei der Schlüssel für mehr Therapietreue zu sein.

Dr. Beate Fessler

Pressekonferenz „100 Jahre subkutane Immuntherapie“ im Rahmen des 6. Deutschen Allergiekongresses. Wiesbaden, 9. September 2011. Veranstalter: Allergopharma, Reinbek

Erhaltungsdosis. In der Per-protocolGruppe waren es sogar $100 \%$ bzw. $98 \%$, erläuterte Prof. Dr. Stefan Zielen, Frankfurt/Main. Die eingangs der Studie sowie nach vier Monaten gemessenen Spiegel der spezifischen Immunglobuline E, G und G4 belegten, dass mit beiden Schemata eine vergleichbar gute Immunresponse erzielt wurde.

Da die Aufdosierung innerhalb von nur drei Wochen außerdem etwa ebenso gut vertragen wurde wie in der doppelten Zeitspanne, dürfte sie laut Zielen angesichts der leichteren Integrierbarkeit in den Alltag besser angenommen werden.

Ute Ayazpoor

Symposium „High-Noon - 100 Jahre Immuntherapie. Ausblick auf die Trends der Zukunft" im Rahmen des 6. Deutschen Allergiekongresses. Wiesbaden, 8 . September 2011. Veranstalter: HAL Allergie, Düsseldorf 